\title{
Towards the elimination of ergot alkaloid biosynthesis genes in Neotyphodium Coenophialum
}

\author{
S. FLOREA ${ }^{1}$, C. MACHADO ${ }^{1}$, D. ZHANG ${ }^{1}$, D.G. PANACCIONE ${ }^{2}$ and C.L. SCHARDL ${ }^{1}$ \\ ${ }^{1}$ University of Kentucky, Department of Plant Pathology, 201 PSB, 1405 Veterans Dr., Lexington, KY 40546-0312, U.S.A. \\ ${ }^{2}$ West Virginia University, Division of Plant and Soil Sciences, 1090 Agricultural Sciences Building, Morgantown WV26506-6108, U.S.A. \\ schardl@uky.edu
}

\begin{abstract}
Neotyphodium coenophialum strain e19 from tall fescue cv. Kentucky 31 carries $d m a W 1$ and $d m a W 2$, two gene homologues that encode dimethylallyltryptophan synthase, the enzyme for the first step in ergot-alkaloid biosynthesis. In our effort to disrupt both homologues and ultimately obtain marker-free mutants, we are using a marker-exchange strategy employing the Cre/ loxP site-specific recombination system. Of 1522 transformants obtained and screened, three were likely dmaW2 disruptants because they gave no PCR product from the wild-type locus, but yielded the larger PCR fragment from the disruption construct. The putative dmaW2-knockouts were also transformed with pKAES186, a plasmid with a cassette containing the cre and ble genes in between loxP sequences. The transformants obtained were screened for the presence of $h p h$, cre and ble genes. The preliminary results indicate a loop-out of the $h p h$ gene. The transformants inoculated into endophyte-free tall fescue preserved their compatibility with the plant. The fungus grown from these plants will be further analysed for the presence of $h p h$, cre and ble genes.
\end{abstract}

Keywords: Cre/LoxP, dimethylallyltryptophan synthase, dmaW, Epichloë, ergot alkaloids, Festuca arundinacea, gene knockouts, Lolium arundinaceum, Neotyphodium coenophialum, tall fescue

\section{Introduction}

In its mutualistic association with the important forage grass, tall fescue (Lolium arundinaceum), the endophyte Neotyphodium coenophialum can have opposing agricultural consequences, improving plant productivity and tolerance to biotic and abiotic stresses, but producing ergot alkaloids associated with livestock toxicosis. One possible way to eliminate this problem is to modify the fungus making it unable to produce ergot alkaloids (Wang et al. 2004). This modification requires introduction of several antibiotic resistance genes, used as selective markers of transformation. The presence of these resistance markers will impede release of plants due to regulatory issues and public concerns. In order to eliminate the resistance genes we chose to use the Cre/loxP system as a method to develop marker free transformants.

Neotyphodium coenophialum has two homologues - dmaWl and dmaW2 - encoding dimethylallyltryptophan synthase (DMAT synthase), the first pathway specific step in ergot alkaloid biosynthesis (Tsai et al. 1995). Several transformation methods were used to replace the native $d m a W 2$ allele with a mutant $d m a W 2$ allele disrupted by the hygromycin resistance gene $(h p h)$ flanked by loxP sites to allow excision by the Cre recombinase. The elimination of the $h p h$ marker by Cre was tested.

\section{Material and Methods}

\section{Strains and culture conditions}

Neotyphodium coenophialum e19 was isolated and maintained as described in Wang et al. (2004). For fungal DNA extraction, mycelial culture was grown on cellophane sheets on potato dextrose agar (PDA) at $21^{\circ} \mathrm{C}$ for 5-6 days. The fungal DNA was from fresh mycelium isolated following the method described by Al-Samarrai and Schmid (2000), and measured using DNA-binding fluorochrome Hoechst 33258 in a Hoefer DyNA Quant 200 fluorometer (Amersham Pharmacia Biotech, San Francisco, California). For fungal transformation, cultures were grown in potato dextrose broth (PDB), shaking at $200 \mathrm{rpm}$ at $21^{\circ} \mathrm{C}$ for 10 days.

\section{Plasmids used for transformation}

Plasmid pKAES174, containing the $5^{\prime}$ and $3^{\prime}$ regions of the dmaW2 locus flanking the loxP-hph-loxP cassette used in transformations, are described in detail elsewhere (Machado 2004). The plasmid pKAES186, was generated by similar techniques, and contains a cassette with the cre (cyclization recombination) and ble (bleomycin and phleomycin resistance) genes between loxP sites.

\section{Fungal transformation}

Protoplasts were prepared as described by Murray et al. (1992), except that mycelium was treated with $35 \mathrm{mg}$ Gluconex, 25 $\mathrm{mg}$ bovine serum albumin, $50 \mathrm{mg}$ Driselase, $8 \mathrm{mg}$ Zymicase, and $50 \mathrm{mg}$ beta-D-glucanase in $10 \mathrm{~mL}$ of osmotic medium. Protoplast yield per $\mathrm{mL}$ averaged $6 \times 10^{7}$. The protoplasts were prepared and transformed by electroporation with SalI-linearized pKAES174 $\left(0.5-5.0 \times 10^{7}\right.$ protoplasts and 4-6 $\mu \mathrm{g}$ DNA in 70 $\mu \mathrm{L}$ ) by the method of Tsai et al. (1992). Alternatively, they were transformed with the linearized plasmid by $\mathrm{Ca}^{2+} /$ polyethylene glycol treatment $\left(0.5-5.0 \times 10^{7}\right.$ protoplasts and $1-2 \mu \mathrm{g}$ DNA in $100 \mu \mathrm{L})$ (Panaccione et al. 2001). The treated protoplasts were then mixed with $7 \mathrm{~mL}$ of complete regeneration medium (CRM) (Panaccione et al. 2001) and poured on top of $20 \mathrm{~mL}$ CRM plates containing hygromycin $\mathrm{B}$ at $48 \mu \mathrm{g} / \mathrm{mL}$. The transformation plates were incubated at $21^{\circ} \mathrm{C}$ for $4-5$ weeks. The fungal colonies were then transferred to PDA with hygromycin B $(48 \mu \mathrm{g} / \mathrm{mL})$ for faster growth, sporulation and single spore isolation.

\section{PCR screening for transformants}

DNA from single spore isolated transformants was extracted by using DNeasy 96 Plant Kit (Qiagen, Valencia, California). PCR amplification was carried out using several sets of primers specific for $d m a W-2$ :

dmaWe19copy2.1d (5'-AGAAACAGACAGGGCTATTC-3'), dmaWe19copy2.5u (5'-CTCGCCGGCATGCGTCAAAA-3'), dmaW144.15d (5'-CGAATGTAGATTACAATGGG-3'), and dmaWcopy2.3u (5'-GCAGTTTGGAGTATCTTTAG-3'),

as well as primers specific for the hygromycin gene:

HYG HY1 (5'-CGAAGAATCTCGTGCTTCAGCTTCGAT GTA-3'), and

HYG_YG1 (5'- CCATTGTCCGTCAGGACATTGTTG-3').

Oligonucleotide primers were from Integrated DNA Technologies Inc. (Coralville, Iowa). Each $25 \mu \mathrm{L}$ PCR reaction mixture included $1.25 \mathrm{mM}$ each dNTP, $5 \mu \mathrm{M}$ each of the two primers, 1X PCR buffer, $2 \mu \mathrm{L}$ DNA template extract and 1.25 units of AmpliTaq Gold DNA polymerase (Applied Biosystems, Roche, 
Branchburg, New Jersey). The reaction mixtures were held at $95^{\circ} \mathrm{C}$ for $9 \mathrm{~min}$, and then subjected to 35 cycles of the following program: $94^{\circ} \mathrm{C}$ for $30 \mathrm{sec}, 61^{\circ} \mathrm{C}$ for $35 \mathrm{sec}, 72^{\circ} \mathrm{C}$ for $3 \mathrm{~min} 10 \mathrm{sec}$.

The plasmid pKAES186, containing a cassette with cre and ble genes between loxP sites was introduced into the dmaW2 knockout mutant. Isolation of the protoplasts from the $d m a W 2$ knockout mutant was described above. The protoplasts were transformed by the PEG method (Panaccione et al. 2001). Transformants were selected on CRM plates containing $25 \mu \mathrm{g} / \mathrm{mL}$ phleomycin and screened by PCR with primer set dmaWe19copy $2.1 \mathrm{~d}$ and dmaWe19copy2.5u specific for $d m a W 2$, a set of primers specific for the $h p h$ gene, HYG_HY1 and HYG_YG1 as well as the following primers to detect $\bar{b} l e$ and cre:

ble seq1 pKAES183: (5'- GAAGCTTCACCTGTCTCTCG-3'), seq15out ble(u) (5'-CTTCGCATCTGGGCAGATGATG-3'), cre (d)Cre(-) (5'-TCCAATTTACTGACCGTA-3'), and (u)Cre-Xbal (5'-CATGTCTAGACTAATCGCCATCTTCCAG CAGGCG-3').

\section{Seedling inoculations}

Tall fescue seedlings were inoculated as described by Latch and Christensen (1985). 'Kentucky 31' endophyte free seeds were surface sterilised and germinated on water agar for 4-5 days. The fungal mycelium was grown on PDA cellophane plates. The seedlings were wounded at or near the shoot apical meristem, and fungal mycelium was placed inside the wound. After inoculation, the seedlings were incubated at $21^{\circ} \mathrm{C}$ for 5 days in the dark and then in the light for 4-6 days. Then the seedlings were planted in soil and placed in the greenhouse. At the three-tiller stage, the seedlings were analysed for the presence of endophyte by tissue-print-immunoblot (TPIB) as described by Gwinn et al. (1991). The preparation of $N$. coenophialum antigen and production of polyclonal rabbit antibodies against it are described by An et al. (1993).

\section{Results}

Homologous recombination in N. coenophialum e19

The plasmid pKAES174 contained a $341 \mathrm{bp}$ deletion from the dmaW2 coding region (starting from the ATG initiation codon), which was replaced by the $h p h$ gene flanked by loxP sites. This plasmid was introduced into $N$. coenophialum e19 by electroporation and polyethylene glycol (PEG) transformation. The 1522 transformants obtained were screened by PCR for homologous recombination with $d m a W 2$-specific primers. These were designed to amplify two different fragments: a $1.5 \mathrm{~kb}$ fragment specific for wild- type and a $2.5 \mathrm{~kb}$ fragment from the dmaW2 mutant. The presence of only the $2.5 \mathrm{~kb}$ band indicated that a recombination event took place and the $2.5 \mathrm{~kb}$ fragment replaced the 1.5 wild-type fragment (Fig. 1). In each PCR screen, wild-type $N$. coenophialum was used as a positive control, and the negative control was $N$. uncinatum (an endophyte of meadow fescue), which lacks dmaW. Of 1522 transformants obtained and screened, three were likely $d m a W 2$ disruptants because they gave no PCR product from the wild-type locus, but yielded the fragment from the disruption construct (Fig. 2). Two of these knockout mutants (dmaW2-ko) were designated e7133 and e7134, and further investigated.

\section{Introduction of transformants into tall fescue cultivar Kentucky 31}

The dmaW2-ko mutants e7133 and e7134, an ectopic transformant, and wild-type $N$. coenophialum e19 were introduced into 'Kentucky 31' seedlings. Seedling infection frequencies for the e7133 and e7134 were higher (67\%) than those for ectopic transformants $(27 \%)$ and for the wild-type $(54 \%)$, but it is unknown if there was a significant difference between these values.

Figure 1 Map of the dmaW2 locus before (A) and after (B) marker-exchange gene disruption, and after subsequent Cre-mediated loss of $h p h$ (C). A primer set, indicated as two arrows in each panel, was used for PCR-amplification of a $1.5 \mathrm{~kb}$ fragment from wt dmaW2 (A), a $2.5 \mathrm{~kb}$ fragment from dmaW2-ko with loxP-hph-loxP (B), and a $1.2 \mathrm{~kb}$ fragment from dmaW2-ko after elimination of $h p h(\mathrm{C})$.

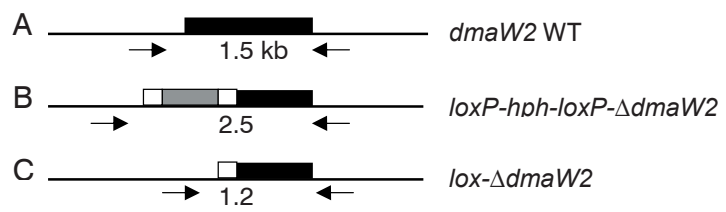

Figure 2 PCR screening for homologous recombinants in which mutated dmaW2 substituted for the wild type gene. PCR with primer sets indicated in Fig. 1 amplified a $1.5 \mathrm{~kb}$ fragment from wt dmaW2 or a $2.5 \mathrm{~kb}$ fragment from dmaW2-ko with loxP-hph-loxP (ko). Nc (N. coenophialum) as positive control, $\mathrm{Nu}$ ( $\mathrm{N}$. uncinatum) as negative control, Ec- ectopic transformant M- 1 kb ladder size marker.

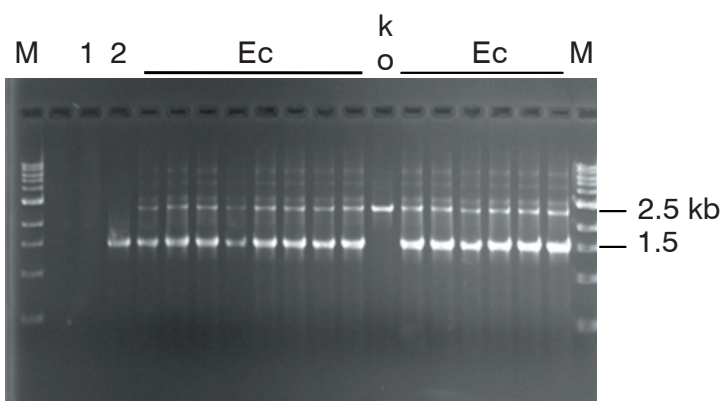

Figure 3 PCR analysis indicating elimination of $h p h$ by Cre recombinase: A PCR test for $N$. coenophialum dmaW2-ko transformed with pKAES186. The dmaW2-ko transformed with pKAES186 (e7135) has lost $h p h$, as indicated by the $1.2 \mathrm{~kb}$ PCR product. PCR from $N$. coenophialum wt (Nc) DNA amplified a $1.5 \mathrm{~kb}$ fragment. PCR from dmaW2-ko (e7133) amplified a $2.5 \mathrm{~kb}$ fragment. $\mathrm{M}$ is $1 \mathrm{~kb}$ marker, 1 ( $N$. uncinatum DNA) and 2 (water) are the negative controls.

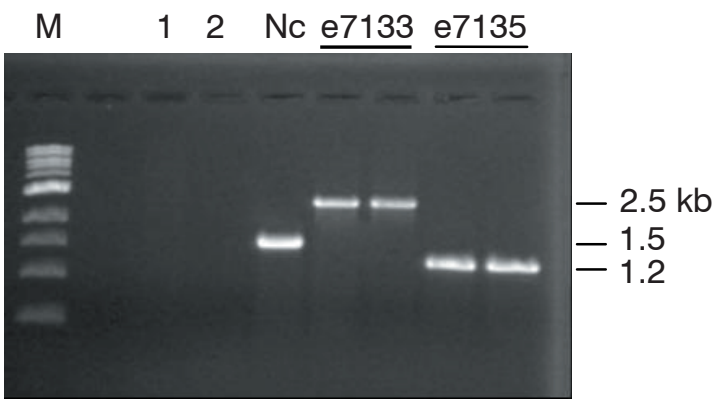




\section{Elimination of $\boldsymbol{h p h}$ in $\mathbf{d m a W 2 - k o}$}

The dmaW2-ko construct has $h p h$ flanked by loxP sites, which are the substrate for the activity of Cre recombinase. The dmaW2-ko mutant e7133 was transformed with pKAES186 carrying a cassette containing ble and cre genes flanked by the loxP sites. The plasmid was designed with the cre gene following an inducible promoter (lolC from $N$. uncinatum), which should cause cre expression in planta. Expression of cre would determine the excision of sequences located between loxP sites, namely, $h p h$, cre and ble. If the $h p h$ gene is eliminated by Cre, a PCR test would result in a $1.2 \mathrm{~kb}$ PCR fragment, compared to the $2.5 \mathrm{~kb}$ fragment from dmaW2-ko and $1.5 \mathrm{~kb}$ fragment in the wildtype $d m a W 2$ (Fig. 1). The transformants were screened by PCR for the construct integration and presence of cre and ble genes. Additional PCR screening was done to verify the elimination of $h p h$ with the same set of primers used for dmaW2-ko screening as well as the $h p h$ gene. The preliminary results indicated that cre was expressed in culture and excised the $h p h$ gene with the retention of a loxP site (Fig. 3).

\section{Seedling inoculations}

The new transformant designated e 7135 was inoculated into tall fescue seedlings as described above. At the three tiller stage, the seedlings were analysed by TPIB to detect the endophyte. Endophyte-infected plants were obtained, but with a lower frequency $(20 \%)$ than obtained earlier with the dmaW2-ko mutants e7133 and e7134.

\section{Discussion}

The ergot alkaloids produced by $N$. coenophialum are a major problem linked with fescue toxicosis in livestock. Wild-type strain e19 from tall fescue cv. Kentucky 31 harbours $d m a W 1$ and $d m a W 2$, two homologous genes for the enzyme catalysing the first ergot-alkaloid biosynthesis step. Our long term goal is to have a modified strain of $N$. coenophialum that does not produce ergot alkaloids and is useful for field purposes. The presence of a foreign gene in the modified $N$. coenophialum could be a public concern. For the elimination of these genes the Cre/loxP sitespecific recombination system was used. The function of Cre in $N$. coenophialum ATCC62374 was tested by Machado (2004). The results showed that the system is efficient, but the modified strain lost its compatibility with the plant. One possible explanation for the loss of compatibility might be repeated subculturing in the laboratory. This was not the case with $N$. coenophialum strain e19 used in this study. The strain is less amenable for laboratory work, but the transformation did not affect its compatibility with the plant. The seedling infection frequency for the dmaW2-ko mutants was $67 \%$, ectopic transformants gave $27 \%$ infection, and for the wild-type we obtained $54 \%$ infection. These high values are surprising, compared to the usual frequency $(15-25 \%)$ that is obtained with routine inoculations of tall fescue with other $N$. coenophialum isolates.
Our preliminary results indicated that the $h p h$ gene was removed in culture by expression of the Promoter $_{\text {lolC }}$-cre construct. Additional testing of these mutants, after reisolation from the inoculated plants, will indicate whether or not the other foreign genes (ble and cre) could also be eliminated.

\section{ACKNOWLEDGMENTS}

This research was supported by United States Department of Agriculture (USDA) National Research Initiative grants 200135319-10930 and 2005-35318-16184, and by a USDA Specific Cooperative Agreement (grant 200403171013).

\section{REFERENCES}

Al-Samarrai, T.H.; Schmid, J. 2000. A simple method for extraction of fungal genomic DNA. Letters in Applied Microbiology 30: 53-56.

An, Z.-q.; Siegel, M.R.; Hollin, W.; Tsai, H.-F.; Schmidt, D.; Schardl, C.L. 1993. Relationships among non-Acremonium sp. fungal endophytes in five grass species. Applied and Environmental Microbiology 59: 1540-1548.

Gwinn, K.D.; Shepard-Collins, M.H.; Reddick, B.B. 1991. Tissue print-immunoblot: an accurate method for the detection of Acremonium coenophialum in tall fescue. Phytopathology 81 : 747-748.

Latch, G.C.M.; Christensen, M.J. 1985. Artificial infections of grasses with endophytes. Annals of Applied Biology 107: 17-24.

Machado, C. 2004. Studies of ergot alkaloid biosynthesis genes in clavicipitaceous fungi. Ph.D. University of Kentucky, Lexington.

Murray, F.R.; Latch, G.C.M.; Scott, D.B. 1992. Surrogate transformation of perennial ryegrass, Lolium perenne, using genetically modified Acremonium endophyte. Molecular and General Genetics 233: 1-9.

Panaccione, D.G.; Johnson, R.D.; Wang, J.H.; Young, C.A.; Damrongkool, P.; Scott, B.; Schardl, C.L. 2001. Elimination of ergovaline from a grass-Neotyphodium endophyte symbiosis by genetic modification of the endophyte. Proceedings of the National Academy of Sciences of the United States of America 98: 12820-12825.

Tsai, H.F.; Siegel, M.R.; Schardl, C.L. 1992. Transformation of Acremonium coenophialum, a protective fungal symbiont of the grass Festuca arundinacea. Current Genetics 22: 399-406.

Tsai, H.-F.; Wang, H.; Gebler, J.C.; Poulter, C.D.; Schardl, C.L. 1995. The Claviceps purpurea gene encoding dimethylallyltryptophan synthase, the committed step for ergot alkaloid biosynthesis. Biochemical and Biophysical Research Communications 216: 119-125.

Wang, J.; Machado, C.; Panaccione, D.G.; Tsai, H.-F.; Schardl, C.L. 2004. The determinant step in ergot alkaloid biosynthesis by an endophyte of perennial ryegrass. Fungal Genetics and Biology 41: 189-198. 